\title{
Facebook: ¿enemigo o aliado? Las empresas periodísticas españolas valoran su relación con la principal red social del mundo
}

\author{
Antonio Méndez Nieto \\ Universidad de Málaga \\ antmendez@uma.es
}

http://orcid.org/0000-0003-1046-5867

\author{
Agustín Rivera \\ Universidad de Málaga \\ agustinrivera@uma.es \\ http://orcid.org/0000-0003-3100-3610
}

\author{
María Bella Palomo Torres \\ Universidad de Málaga \\ bellapalomo@uma.es
}

https://orcid.org/0000-0003-2228-5716

\section{Facebook: enemy or allied? Spanish media companies value their relationship with the main social network in the world}

\begin{abstract}
RESUMEN
Con casi 2.000 millones de seguidores en el mundo según Internet World Stats, 23 de ellos en España, Facebook hace tiempo que dejó de ser una red social para reunir amigos. Su propio creador acepta ya que también entre sus objetivos figura transformarse en un potente medio de comunicación. De momento, subproductos como Instant Articles y Facebook Live han irrumpido con fuerza y obligado a los principales grupos de comunicación a trabajar para esta gran plataforma de distribución de contenidos. Este artículo profundiza en el impacto de Facebook en los medios de comunicación españoles, cómo influye en sus rutinas, los contenidos y la determinación de la agenda, así como las posibilidades de negocio que genera en una etapa marcada por la incertidumbre. Para ello se ha entre-

vistado a los responsables de participación y medios sociales de ocho de las empresas de comunicación más importantes de España. Los resultados desvelan que para la mayoría, la red supone una amenaza por sus cambios continuos de algoritmo, aunque aceptan el riesgo porque su uso es fundamental para intentar captar audiencia.
\end{abstract}

PALABRAS CLAVE

Periodismo digital, Facebook, Red social, Interacción, Audiencia

\section{ABSTRACT}

Almost 2 billion followers in the world, 23 of them in Spain. According to these data from the Internet World Stats, Facebook has long ceased to be a social network to gather friends. Its own creator confirms that among its objectives figure to become a powerful news media. At the moment, products such as Instant Articles and Facebook Live have burst in and force major communication groups to work for this great content distribution platform. Our article explores the impact that Facebook has on the Spanish media, its routines, content and its influence on the agenda-setting. To reach these goals, we interviewed those responsible for participation and social media in eight of the most relevant communication companies in Spain. Results show that Facebook means a threat due to its continuous algorithm changes in most cases, although media accept the risk because its use is fundamental to attract audience.

\section{KEYWORDS}

Digital Journalism, Facebook, Social

Network, Interactivity, Audience 


\section{Introducción}

Algo realmente dramático sucede en el paisaje de los medios de comunicación y en la industria del periodismo en general. Ocurre de una forma casi imperceptible y sin que se desarrolle un examen público y el debate adecuados. Es el escenario que describe Emily Bell (2016) en su artículo "Facebook is eating the world", sobre la red social creada por Mark Zuckerberg en 2004 originariamente para poner en contacto a estudiantes de la Universidad de Harvard. Con su desarrollo exponencial, en poco más de una década ha logrado captar a través de sus productos a casi 2.000 millones de personas en el mundo, 23 de ellos en España (Internet World Stats, junio 2017).

Díaz Gandásegui (2011) ya señaló que las características de Facebook la convierten en un espacio ideal para la prensa al ser una plataforma de comunicación horizontal y recíproca que la consolida como la red adecuada para frecuentar contactos, crear comunidad y generar fidelidad hacia una marca. Estas funciones constituyen los principales objetivos que se plantean los grupos de comunicación en relación a las audiencias, además del negocio económico si finalmente la relación alcanza el grado de simbiosis.

Afirma Bell (2016) que el ecosistema de noticias ha cambiado más dramáticamente en los cinco años pasados que quizás en cualquier momento de los últimos quinientos. Saltos ligados a una revolución móvil, que van de la realidad virtual y aumentada, a las transmisiones de vídeos en directo, las informaciones artificialmente inteligentes, los programas de edición, la mensajería inmediata, o las 'apps'. Para tratar de alcanzar la rentabilidad económica muchos medios se han visto forzados a dirigirse directamente a Facebook y a sus instant articles, donde el bloqueo de los anuncios es más difícil que en el navegador. Pero los riesgos de confiar los ingresos y el tráfico a un distribuidor externo son muy altos (Bell, 2016). La pérdida de la marca, la falta de datos de las audiencias y la migración de los ingresos publicitarios siguen siendo preocupaciones claves para los editores.

En este contexto Facebook se presenta a su vez como un aliado y como el enemigo público número uno de los medios de comunicación. Apenas pasa un día en el que no haya un artículo negativo sobre el sitio web de medios sociales que está atrayendo a "nuestros" lectores y anunciantes, expone Roy Greenslade (The Guardian, 2016), con un software que bloquea informaciones y anunciantes y que limita la agenda de noticias, aconsejando a los usuarios qué leer. Unas opciones elaboradas por algoritmos que pueden monitorear los intereses de los usuarios y luego "alimentar" lo que considera desean leer, mientras filtran el material que se supone no les interesa y que dejan fuera de la circulación. La influencia de las plataformas sociales es patente en la agenda actual de los medios. Las redes sociales ofrecen incentivos a las organizaciones de noticias para determinados tipos de contenido, como vídeos en directo, o imponen la actividad editorial a través de normas de diseño o explícitamente editoriales, según analizan Bell y Owen (2017).

Eli Pariser, quien fuera entre 2003 y 2008 el director de MoveOn.org, el primer gran movimiento político online, preocupado por los algoritmos de Facebook y Google publicó el libro El filtro burbuja. Cómo la Red decide lo que leemos y lo que pensamos (2017). En su trabajo explica las graves consecuencias que las directrices de estas compañías suponen para la recepción de la información y, en consecuencia, cómo influyen en la forma de pensar. El autor intenta combatir con la web de noticias Upworthy, de la que es cofundador, lo que se denomina 'filtro burbuja', una expresión popular en Estados Unidos para hacer referencia a las restricciones antes citadas. Para él, la clave de los algoritmos es el entretenimiento y éste proporciona más clics que las noticias duras (El Mundo, 2017).

Pero los medios también deben afrontar otros problemas derivados de las estrictas normas de la distribuidora. Contenidos a su juicio violentos o de cualquier índole sexual están vetados para su publicación. La realidad del periodismo puede chocar frontalmente con los deseos de quien establece las reglas porque ostenta el poder. Las advertencias o amonestaciones cuando a los 'censores' les chirrían los contenidos están a la orden del día. El riesgo a perder de golpe la audiencia existe y la renuncia a publicar determinados hechos o suavizarlos, también.

\subsection{El almacén de audiencias}

Estos inconvenientes se trasladan a un segundo plano debido a un dato. Por el volumen de usuarios, Facebook es la red social más importante del mundo y, por tanto, el nicho más amplio que tienen a su disposición los medios de comunicación para intentar captar nuevas audiencias. Paralelamente, esta situación les genera una gran dependencia (Singer, et al., 2011). Hay cibermedios que cifran en un tercio el número de visitas que les reporta Facebook (Somaya, 2014).

El consumo de noticias a través de las redes sociales cada vez es más intenso y hay estudios que demuestran que Facebook es un canal adecuado para la publicación de últimas noticias ya que proporciona una rápida viralidad (Díaz Campo, Segado Boj y Soria, 2015), favorece la participación y la aceptación pública (Coca, 2009).

Estos supuestos contrastan con el comportamiento reflejado en diversos estudios donde se revela la escasa interacción existente en Facebook entre usuarios y medios (Gómez Molina y Ramos del Cano, 2014). No parece que el transcurrir de los años y los avisos constantes hayan conseguido transformar estas rutinas. Pese a la importancia de las redes sociales en la difusión de los contenidos, el personal dedicado a esta actividad en los principales grupos españoles es escaso y a veces solo testimonial. 


\subsection{Una actividad rentable de dudosa calidad}

La principal red social en internet logró un beneficio en el segundo trimestre de 2017 de 3.890 millones de dólares, tras registrar unos ingresos de casi 9.500 millones, un $45 \%$ superiores a los de las mismas fechas el año anterior, fruto del fuerte incremento de la demanda publicitaria derivada del valor de un volumen importante de usuarios con una implicación adictiva.

Tras las pasadas elecciones en Estados Unidos, sin embargo, recibió fuertes acusaciones por lucrarse con anuncios mientras su sistema era incapaz de controlar la divulgación de noticias falsas. La plataforma de Mark Zuckerberg rechazó cualquier responsabilidad en el tráfico de bulos, pero lanzó una declaración con una serie de compromisos para descubrir la desinformación, que, sin embargo, no incluía la verificación de los contenidos que circulan entre los usuarios. A pesar de ello, encargó a un equipo conformado por medios y académicos la creación de un sistema que permitiera discernir entre la verdad y la mentira.

También estas acusaciones provocaron otra reacción sumamente inteligente. A principios de 2017 Facebook suscribió un acuerdo con nueve medios de comunicación de referencia internacional a los que convertía en sus aliados. Entre ellos figuraba el diario El País, que ya había decidido en 2008 que sus contenidos debía explotarlos en esta red social. La iniciativa llamada Facebook Journalism Project pretende estrechar la relación entre Facebook y la industria informativa. "Colaboraremos con las organizaciones informativas para desarrollar productos, aprender de los periodistas sobre cómo ser mejores socios y trabajar con editores y educadores sobre cómo podemos equipar a las personas con el conocimiento que necesitan para ser lectores informados en la era digital", añade Facebook en un comunicado publicado en España por El País (Seco, 2017).

Al lanzar proyectos informativos con El País, "la mayor red social del planeta distinguirá y destacará información contrastada y veraz, a la vez que experimentará con formatos innovadores", resaltaba el buque insignia del grupo Prisa. Una de las novedades consiste en ofrecer cada mañana a los usuarios una selección de noticias elaboradas y editadas por periodistas. La apuesta informativa está diseñada exclusivamente para consumo por móvil y consta de cinco contenidos especiales al día en el newsfeed del usuario. Pero con unas condiciones muy ventajosas para la plataforma social, según listaba El País (2017):

- 1. Para ver nuestra apuesta diaria, tienes que ser seguidor de El País en Facebook.

- 2. El contenido aparecerá en tu hilo de noticias a las 6 de la mañana y se adaptará a tu hora local.
- 3. Solo podrás verlo en el móvil. Las noticias tienen formato de instant articles, un sistema de descarga rápida de Facebook para teléfonos inteligentes que El País usa desde principios de 2016. Como instant articles, verás tanto los textos como las fotos y los vídeos de forma instantánea.

\subsection{Los estudios subrayan la ausencia de diálogo con la audiencia}

Son numerosos los trabajos que se han acercado en los últimos años a la realidad de Facebook desde la perspectiva de los medios de comunicación, así que sólo citaremos los más próximos a la perspectiva de este trabajo. La presencia de los medios en los perfiles de Facebook se ha tratado tanto en publicaciones profesionales (Coca, 2009; San Juan, 2012) como en otras de índole científica. Un buen ejemplo de las primeras es el informe Facebook Top Newspaper, Social Rating and Ranking, publicado en 2012 por la consultora italiana Innova et Bella, en el que se explora cómo algunas de las cabeceras más importantes del mundo aprovechan sus perfiles en esta red social (Merino Bobillo, et al., 2013).

En el ámbito español, cabe resaltar el estudio de José Manuel Noguera Vivo (2010), quien ofrece una plantilla de análisis de contenidos de cibermedios asociados a cabeceras en papel, regionales y nacionales, que tienen perfil en Facebook. Su trabajo concluye que los diarios utilizan esta comunidad virtual como un agregador de noticias, pero no aprovechan suficientemente las facilidades interactivas para fines periodísticos, algo que como veremos más tarde no ha variado sustancialmente en la actualidad.

Aunque cualquier estudio cuantitativo sobre Facebook pierde vigencia a gran velocidad, merece la pena apuntar el de Merino Bobillo et al. (2013), quienes analizan los siete periódicos con mayor audiencia en España entre octubre y diciembre de 2012. Señalan que la interacción generada mediante las opciones básicas de Facebook ('me gusta', 'comentar' y 'compartir') constituye una fuente de información valiosa respecto al grado de implicación de los lectores con la noticia y con el medio. Por eso, los periódicos que mejor aprovechan las posibilidades de esta red social abogan por aumentar el valor de los contactos más allá del simple 'me gusta'. Pero el trabajo también concluía que los diarios analizados utilizan Facebook un día de cada dos, y este uso se encuentra más desarrollado en las cabeceras deportivas tanto por el número de días en los que publican como por la cantidad de noticias que generan.

Asimismo, es preciso mencionar la investigación de María Remedios Guirado Zamora (2012), sobre los diarios locales de Andalucía y los nacionales que se distribuyen en esa comunidad autónoma. Su análisis demuestra una alta participación de los seguidores de esos medios y confirma el empleo de Facebook como herramienta de difusión de contenidos. 
En un entorno también próximo, González Molina y Ramos del Cano (2013) abordaron un análisis comparativo de los usos periodísticos de Facebook y Twitter en 41 medios de comunicación europeos. La principal conclusión a la que llegaron fue que la función más habitual que los medios europeos dan a sus perfiles en Facebook y Twitter era también difundir información. Así, de los 2.274 mensajes que analizaron, un 81,09\% se encuadraba en esta categoría, mientras que un 13,76\% era promoción y un 5,10\% diálogos con el usuario. En esa misma línea se encuentra el trabajo de Palomo y Meso (2014), que califican de "utopía" que en Facebook se pueda hablar de diálogo entre la audiencia y el medio. Para llegar a esa conclusión estudiaron durante una semana las entradas y comentarios en los muros de El País, The Guardian y The New York Times y observaron que los diarios no reaccionaban a las demandas de las audiencias. En definitiva, la interacción con el usuario es una asignatura que continúa pendiente en la mayor parte de los grupos de comunicación.

\section{Objetivos}

La novedad de esta investigación reside en sus objetivos, ya que pretende trasladar la visión interna de los encargados de fabricar, posicionar y explotar los contenidos informativos en la red. Este objetivo general permite conocer qué ventajas futuras o presentes proporciona esa colaboración y qué riesgos corre la propia marca al aceptar las reglas que impone la plataforma. Además, el artículo persigue constatar cuál es la importancia real de Facebook para los grupos, cuál es el nivel de confianza y de recelo que genera, y conocer cómo afrontan este desafío.

Divulgar las rutinas diarias de las empresas periodísticas españolas en su relación con esta gran red social y describir qué fórmulas desarrollan para incrementar sus audiencias a través de la misma o cómo afrontan estar sujetos a unas reglas cambiantes, resulta relevante cuando decididamente Facebook ya no se conforma con la distribución de contenidos, sino que ha decidido jugar también un papel activo en el ámbito de la información.

\section{Metodología}

Para alcanzar estos objetivos se ha planteado un enfoque meteorológico cualitativo, basado en la entrevista en profundidad semi-estructurada con ocho responsables de participación y medios sociales de tres medios nacionales ( $E l$ País, El Mundo, Abc), cuatro nativos digitales (El Confidencial, eldiario.es, The Huffington Post, El Español) y un grupo de medios locales (Grupo Joly), cuyas páginas comunes se comportan como un grupo regional.

Las entrevistas se realizaron en las redacciones, entre enero y marzo de 2017. Todos los contactos fueron personales, excepto

\begin{tabular}{|l|l|}
\hline Entrevistados & Perfil \\
\hline Ander Oliden & $\begin{array}{l}\text { Editor de Medios Socia- } \\
\text { les de Eldiario.es }\end{array}$ \\
\hline $\begin{array}{l}\text { Ángela Rodríguez Bona- } \\
\text { chera y Patricia Morales }\end{array}$ & $\begin{array}{l}\text { Responsables de Redes } \\
\text { Sociales de El Español }\end{array}$ \\
\hline Fermín Elizari & $\begin{array}{l}\text { Editor de Medios Socia- } \\
\text { les de El Mundo }\end{array}$ \\
\hline Guillermo Rodríguez & $\begin{array}{l}\text { Subdirector de Huffing- } \\
\text { ton Post en español }\end{array}$ \\
\hline Javier Corcuera & $\begin{array}{l}\text { Editor de Medios Socia- } \\
\text { les de Abc.es }\end{array}$ \\
\hline Jesús Ollero & $\begin{array}{l}\text { Responsable de Partici- } \\
\text { pación del Grupo Joly }\end{array}$ \\
\hline José Manuel Rodríguez & $\begin{array}{l}\text { Editor de Medios Socia- } \\
\text { les de El Confidencial }\end{array}$ \\
\hline Pilar Millán & $\begin{array}{l}\text { Directora de desarrollo de } \\
\text { audiencias de Prisa Noticas }\end{array}$ \\
\hline
\end{tabular}

Tabla 1. Responsables de participación y redes sociales entrevistados. Elaboración propia.

en el caso del responsable de medios sociales del grupo Joly, que se grabó telefónicamente. A partir de un cuestionario prediseñado, que partía de un par de cuestiones generales previas sobre las redes sociales, se profundizó durante el diálogo en aquellos aspectos de más interés para esta investigación para obtener una mayor precisión en las respuestas. Para estructurar el artículo, tras inferir los resultados y ver los grados de coincidencia, se estableció un esquema fundamental de exposición de los mismos, modulado por los distintos grados de influencia, respecto a las siguientes variables del binomio red-medio: agenda, audiencia, rutina, marca, contenidos y negocio.

\begin{tabular}{|c|}
\hline Cues \\
\hline $\begin{array}{l}\text { ¿Hay personal específico dedicado a redes sociales? En caso } \\
\text { afirmativo, ¿a cuáles y cuántas personas están implicadas? }\end{array}$ \\
\hline $\begin{array}{l}\text { Ordene las redes sociales en función de la importancia otor- } \\
\text { gada por su medio }\end{array}$ \\
\hline $\begin{array}{l}\text { ¿Obligan o recomiendan la presencia de sus periodistas en } \\
\text { las redes? }\end{array}$ \\
\hline ¿Hay normativa interna sobre redes sociales? \\
\hline $\begin{array}{l}\text { ¿Programan contenidos durante la jornada para ser } \\
\text { publicados automáticamente en redes sociales? }\end{array}$ \\
\hline ¿Se fijan objetivos de audiencia? \\
\hline $\begin{array}{l}\text { ¿Hay interlocución con los usuarios? En caso afirmativo, ¿de } \\
\text { qué tipo? ¿Quién se encarga de la moderación? }\end{array}$ \\
\hline $\begin{array}{l}\text { ¿Influyen los mensajes de los usuarios en la agenda del } \\
\text { medio? }\end{array}$ \\
\hline $\begin{array}{l}\text { ¿Hay recomendaciones o estrategias internas para la } \\
\text { selección y publicación de soft y hard news? }\end{array}$ \\
\hline ¿Qué porcentaje de audiencia visita al medio vía de Facebook? \\
\hline $\begin{array}{l}\text { ¿Cuántos post al día difunden en Facebook y con qué perio- } \\
\text { dicidad? }\end{array}$ \\
\hline $\begin{array}{l}\text { ¿Participan en Facebook Live? ¿Con qué periodicidad? ¿Les } \\
\text { resulta rentable? }\end{array}$ \\
\hline ¿Pagan por promocionar contenidos en Facebook? \\
\hline n se debe temer más: a Facebook o a 0 \\
\hline
\end{tabular}

Tabla 2. Preguntas de la entrevista. Elaboración propia. 


\section{Resultados}

¿Aliado o adversario? Posiblemente no es necesario decantarse por una de las dos opciones o, mejor aún, podría decirse que ambas son compatibles. Los principales grupos de comunicación de España son conscientes de la influencia de Facebook en sus audiencias por lo que no albergan dudas de la necesidad de su presencia en la gran plataforma social. Pero su imprevisible comportamiento se traduce en una clara desconfianza. La red social ya no es un simple canal para distribuir contenidos, sino que es un nuevo espacio de consumo de productos informativos que aleja a los medios del origen inicial de esa mutua colaboración: el aumento del tráfico para sus webs.

La posibilidad que ofrece Facebook Live permite formular una cuestión que se preguntan algunos responsables de los principales grupos españoles de comunicación: Finalmente, ¿para quién trabaja el periodista que los hace: para su medio o en realidad para Facebook, como un apéndice más de esta gran multinacional?

Su rápida viralidad y la fidelidad que muestran sus audiencias, además de la futura posibilidad de introducir publicidad en los vídeos e implementar las fórmulas de negocio son elementos determinantes en el lado positivo. El riesgo de diluirse como una marca blanda y la imposibilidad de conocer con antelación el algoritmo de la red son algunos de los graves inconvenientes que afrontan los medios. Además de las estrictas reglas sobre algunos contenidos que, en ocasiones, limitan el ejercicio del periodismo.

Todos los responsables de medios sociales consultados para este trabajo señalan que su primer objetivo es Facebook. "Es Dios", llega a asegurar Guillermo Rodríguez, subdirector de Huffington Post en español. "Todos somos conscientes del poder que tiene, sobre todo porque se está comiendo a todos", recalca Fermin Elizari, editor de medios sociales del diario El Mundo. Facebook es la red más rentable a la hora de atraer visitas, aunque dediquen más tiempo a Twitter, como confiesan Ángela Rodríguez Bonachera y Patricia Morales, responsables de redes en El Español, porque "al ser más realtime tienes que estar mucho más encima. En Facebook lo dejas ahí y que crezca", explica.

Para Javier Corcuera, editor de medios sociales de Abc.es, el número uno, el top, es Facebook sin ninguna duda por el tráfico que genera. Además, han detectado que hay una parte de la población que se informa específicamente a través de las redes sociales. No obstante, sitúa al mismo nivel a Twitter, porque figuran todos los periodistas. Pero quien está más convencido de la fuerza de Facebook es el grupo Prisa, y concretamente El País. Para Pilar Millán, directora de desarrollo de audiencias de Prisa Noticias, "nuestra prioridad es Facebook evidentemente por la cantidad de audiencia" y agrega que las redes sociales para el grupo no son plataformas sólo de distribución, sino que ya se ha convertido con los instant articles de Facebook en un gran espacio de consumo de contenidos. Razón por la que entiende que las redes ya no son simplemente plataformas de distribución sino también de consumo.

Jesús Ollero, responsable de participación y redes del grupo Joly en el momento en que se formalizó esta entrevista (febrero de 2017), explica que ahora se centran en Facebook para intentar reconducir una carencia que tenían de comunidad. Las demás redes "son secundarias" en comparación, admite. Para José Manuel Rodríguez, editor de medios sociales de El Confidencial, existe un triángulo imaginario que induce a la participación en las redes sociales con tres vértices: el desarrollo de la marca, el de la audiencia y el del negocio. Todas las respuestas obtenidas en este trabajo inciden en que son esos los fines que los grupos de comunicación españoles persiguen, sobre todo, en Facebook.

Pese a que casi todos los entrevistados admiten los elevadísimos porcentajes de audiencia que les reporta su presencia en la red creada por Zuckerberg, en algún caso incluso del 85\% del tráfico global que les llega por esas vías de procedencia, se aprecia, sin embargo, un exceso de voluntarismo y un gran desconocimiento a la hora de afrontar el fenómeno, con la excepción de El País. Los frentes abiertos en la actualidad para los grandes grupos de la prensa en España son tan amplios que son imposibles de atender con los medios deseados. $\mathrm{Ni}$ siquiera Facebook ha 'obligado' a estos grupos de comunicación a destinarle un personal específico.

\section{1. ¿Socio o enemigo? Reflexiones sobre una obligada dicotomía}

Si no miedo o temor, como se suele decir de los adversarios en las competiciones deportivas, mucho respeto. El sentimiento general de la mayoría de los grupos de comunicación consultados linda con esa percepción, con dos excepciones: El País y El Mundo. La pregunta directa que se formalizó inquirió sobre los dos gigantes: Google y Facebook y la elección de cuál de ellos suscita en la actualidad mayor temor. La segunda empresa ha adquirido la dimensión que obtuvo la primera a principio de este siglo, cuando cualquier cuestión que se plantease en el mundo significaba que había que consultar indefectiblemente su buscador.

José Manuel Rodríguez, editor de medios sociales de El Confidencial, profundiza en su respuesta:

Un amigo-enemigo porque cuando tú hablas de Google y Facebook estás hablando de dos empresas que te quieren echar una mano. Pero la mano, ¿dónde te la quieren echar? ¿Te la quieren echar en la cabeza para hundirte, en el hombro para apoyarte o en el bolsillo para quitarte la pasta? Esa es la pregunta. A lo largo de todo el día tú pue- 
des tener las tres sensaciones... Nos vemos obligados a cohabitar con ellos, pero sí que es verdad que tenemos que ser muy cuidadosos con lo que hacemos para que no nos coman la tostada, pero tampoco para dejar pasar oportunidades que son importantes. Sobre todo, porque en la medida tienen una gran capilaridad son una gran oportunidad.

El responsable de este diario nativo digital define que el objetivo es conseguir lectores y evitar que la audiencia identifique al medio como una marca blanca más de Facebook. Y relativiza los números. Asegura que la red cuenta con 25 millones de seguidores en España y apenas un 3\% del total está suscrita o sigue a El País, el medio más conocido de España, por lo que duda que el público forme parte de la plataforma con el ánimo de obtener información. Desde El Español se describe directamente a Facebook como una plataforma "opaca" y se la teme incluso más que a Google por los efectos que sobre el tráfico tienen sus decisiones._Jesús Ollero, del Grupo Joly, también se inclina por el peligro que representa la gran plataforma de distribución en oposición al agregador, pero principalmente porque está ofreciendo la posibilidad, y hay medios que están aceptándola, de acceder al contenido de una página web sin tener que entrar en la misma gracias a los instant articles que han desarrollado, comenta. "Google seguramente no tiene la fidelidad que puedes conseguir en Facebook, pero necesariamente te va a desviar tráfico".

Ander Oliden, editor de medios sociales de Eldiario.es, también abunda, como los anteriores, en las muchas prevenciones que suscita esta red. Y está convencido de que no va a desaparecer. Admite que se han dado casos con navegadores que sucumbieron, como AltaVista y Yahoo!, "que era un gigante está ahora hundido", pero por lo menos durante un tiempo relativamente prolongado "vamos a convivir con Facebook". Narra que Facebook le proporciona bastantes disgustos. No quiere hablar de temor, pero sí de peligro por su algoritmo y por las tendencias que marca. Explica que un medio puede realizar un enorme esfuerzo, incluso económico para construir una comunidad y venírsele abajo con un simple cambio.

Hubo muchas empresas que crearon comunidades pagadas en Facebook, cambiaron los algoritmos y esas comunidades pagadas a día de hoy no ven información y entonces Facebook decidió que tenías que pagar para promocionar cada uno de los contenidos que hacías. Y ¿cuál será la siguiente frontera? Pues la que decida Facebook, de la misma forma que cuando Zuckerberg dice: jla tendencia del año que viene va a ser el vídeo en directo! Pues si tu algoritmo favorece que los contenidos en Facebook Live sean con muchísima diferencia los que más alcance obtengan, pues lógicamente será la tendencia.
Guillermo Rodríguez, subdirector del Huffington Post en español insiste en los algoritmos, que son el elemento del funcionamiento interno de la gran multinacional que más desconciertos provoca. Admite su sospecha de que los modifican constantemente y compara lo que sucede con el antecedente de Google en 2003. Entonces se dedicó personal específico para posicionar al medio y asegura que ahora se hace lo mismo con la red, con el riesgo de que su propietario pueda tomar la decisión algún día de dejar de publicar los medios. Pilar Millán, directora de desarrollo de audiencias de Prisa Noticias, opina que ni Google ni Facebook deben provocar resquemor porque sería como temer al desarrollo de la audiencia y significaría una visión muy poco digital de la realidad. Entiende que el objetivo que se debe plantear desde la óptica del grupo de prensa es cómo conseguir convertirlos en aliados y cómo lograr que conjuntamente pueda desarrollar productos y servicios adecuados para esa plataforma para hacer que el usuario "consuma tu contenido, entienda tus valores y siga enganchado a tu información".

Facebook anunció hace poco un acuerdo. Admitió Mark Zuckerberg que es una empresa de medios. Hasta entonces todo habían sido negativas, ellos no querían ser reconocidos como un medio de comunicación. Eso ha cambiado. Luego enviaron un comunicado para explicar un gran acuerdo, Facebook for Journalist, que tiene tres patas: desarrollo de productos y servicios, herramientas de analítica para periodistas y, la última, detección de noticias falsas. Nosotros llevamos unos meses con una relación con Facebook, mucho más en el sentido de vamos a trabajar juntos, vamos a entender mejor a la audiencia, vamos a desarrollar productos conjuntamente, vamos a entendernos y de momento todo ha sido muy positivo.

Aun así, advierte la responsable de Prisa que Facebook tiene un problema con las analíticas, en general, y que, en ese sentido, no tiene muy controlada la necesidad de datos que tiene un medio de comunicación que es muy diferente al de una marca de otro sector o una marca de consumo. La plataforma necesita poco dato y el periódico un ingente volumen y "mucha información y además generamos muchísimo volumen de datos al propio Facebook". De ahí deduce que ese aspecto está mal gestionado, aunque lo atribuye a que en el fondo se trata de una empresa muy joven que ha tenido un desarrollo exponencial en muy corto periodo de tiempo: "La parte analítica para mi es el caballo de Troya de Facebook", sintetiza Millán.

En la misma línea de Prisa, desde el diario El Mundo, Fermín Elizari, defiende que los medios no deben de temer a alguien y opina que Facebook "lo ha hecho muy bien" y es esencial para acceder a una gran audiencia, por lo que no hay alternativa. Si la discrepancia es insalvable "pues no pongas tu contenido en Facebook, es lo mismo que antes pasaba con Google". Y está 
convencido de que los contenidos que aportan los medios son "vitales" para las dos citadas compañías. Y acentúa con datos la importancia de la red, que según su opinión en lo que finalmente se fijan los anunciantes: El Mundo tiene 2'6 millones de seguidores en Twitter y en Facebook 1'8 millones, pero ésta le aporta muchísimo más tráfico y mayor interacción; incide en que aquí la audiencia activa es "clave". Pero a la vez realiza una defensa de la audiencia directa:

¿Qué es más valioso: el usuario que va directamente a El Mundo o el que va a ElMundo.es a través de una noticia de Facebook? Es mucho más valioso el tráfico directo. Muchas veces nos ponemos todos como locos para aumentar nuestro tráfico de redes. Igual prefiero ser el que tenga más tráfico directo porque es la gente más fiel. Creo que alguien que se registre o que comente dentro del artículo implica para empezar que se ha podido leer todo el artículo entero, porque muchas veces en Facebook la mitad de los comentarios o muchos comentarios son de gente que igual ni ha clicado.

\subsection{La agenda y las restricciones por prescripción}

Un post cada media hora. Ese es el ritmo de publicación que la mayor parte de los grupos de comunicación dedican al día a Facebook. En algunos casos como El País calculan que suman unas 70 comunicaciones cada jornada destinadas a la red social. A diferencia de Twitter, que se considera más cronológica, en Facebook no se puede "saturar al usuario o al lector con demasiados temas en poco tiempo", opina Ollero, del Grupo Joly. Rodríguez, de El Confidencial cree que las urgencias en Facebook son innecesarias y valora la posibilidad de establecer horarios para combinar soft news y hard news porque predominan otros factores antes que el cuándo se ha publicado. En Facebook da igual a qué hora se publique un contenido porque es "una máquina de resultados", asegura el responsable de El Mundo, quien indica que la red puede crear una información como una bola de nieve que se prolonga durante horas. Agrega, además, que Facebook piensa sólo en el usuario y si detecta que algo le va a interesar da igual que se haya publicado hace cinco minutos otro asunto. Por ello Abc. es reserva las breaking news para Facebook.

Las redes sociales y la participación han adquirido importancia en los últimos años para los medios, hasta el punto de destinar personal específico a estos ámbitos. De una a diez personas. El primer dato corresponde al grupo Joly, y el último es el que facilita el diario El País, que al tener sedes en Latinoamérica suma a su personal en España otras dos personas en México y una tercera en Brasil para mantener la actividad durante las 24 horas del día. El Mundo emplea a cinco redactores y dos becarios. Pero, en general, son tres o cuatro las personas destinadas a este área y nadie específicamente se ocupa de Facebook pese a su importancia. Fermín Elizari cree que su departamento sería prescindible si los redactores supieran vender bien sus piezas en redes sociales. Ésa es la fórmula escogida por el Huffington Post. Sin un community manager, son los redactores los que vuelcan sus informaciones en las redes. Con dos secciones genéricas, hard news y soft news, la única obligación es que haya suficiente contenido y el apropiado para la red, pero con organización, lo que incluye una escaleta de horarios.

En cuanto a cómo pueden aprovechar los medios las ventajas que pueden proporcionar las redes sociales, también hay disparidad de criterios incluso dentro del mismo grupo editorial. Así, en Prisa existe una guía explicativa para El País con unas 150 páginas de donde se desmenuzan todas las posibilidades, mientras el subdirector del Huffington Post en español aplica la lógica. Los porcentajes tampoco son homogéneos al valorar el impacto de Facebook en las audiencias. Para El País representa el 85\% de las visitas vía redes sociales; El Español cifra el porcentaje en algo más del 80\%. En Abc, Facebook les aporta el $90 \%$ de la audiencia que le llega vía redes sociales y que supone entre el $83 \%$ y el $86 \%$ del total que penetra vía móvil. El retraso del grupo Joly en esta materia es importante, y estima que los medios sociales sólo le aportan el 16\% de sus audiencias en internet. El Confidencial, un medio nativo digital, rebaja hasta el 10\% la aportación de esta plataforma al cómputo global de sus audiencias.

Respecto a la formación, también existe un amplio contraste entre medios. Abc sí imparte algunos cursos sobre redes sociales a la redacción, sobre todo por secciones. Esta red, para este diario tiene un gran potencial para áreas como ciencia o historia. En El Español, los responsables se plantean realizar un curso sobre Facebook para los redactores. En El País sí se ha facilitado formación específica a la redacción y recomendado y ayudado a los periodistas a que se abran perfil en esta red, dada su preferencia por Twiter. Para esta tarea contaron con la colaboración de un equipo de Facebook. Las secciones poseen páginas en esta red, en un proceso que califican de "evangelización" y que se desarrolló en 2015. El editor de medios sociales de Abc.es afirma que hay periódicos que sí están utilizando las páginas de Facebook de los propios periodistas como un método de difusión. "Nosotros no estamos en ese punto", aclara Javier Corcuera. Defiende que se ha superado la desconfianza de la redacción a las redes: "Han visto que Facebook no va a jubilar a nadie" y afirma que cada vez se le aprecian más ventajas para el trabajo periodístico y eso gusta en la redacción.

Otro asunto es la interacción. Los comentarios "nos cuesta mucho mirarlos", dicen desde El Español: "Puede haber 300 en una publicación y miramos la conversación para ver "si se lía". En el Huffington los usuarios tienen que registrarse en Facebook para poder dejar sus comentarios. En El País los comentarios diarios se acercan al millar y por ello una empresa externa se encarga de la moderación. La falta de 
medios impide prestar atención a esos extensos diálogos y en algunos casos, incluso se aboga por no intervenir.

Los medios en general no admiten que Facebook posea un impacto apreciable en la conformación de su agenda, aunque El País afirma que a veces reciben comunicaciones o alertas de usuarios que se utilizan luego para elaborar noticias. El Huffington, por el contrario, es el único que asegura que condiciona la agenda "totalmente". El tráfico es el principal objetivo de los grupos en su acercamiento a Facebook, al menos hasta este momento, además de afianzar la imagen de marca y explorar posibilidades futuras de negocio. Y la viralidad es un gran atractivo de la red, con sus condicionantes. Juan Manuel Rodríguez de El Confidencial define el círculo virtuoso. Describe que un hito en redes rara vez se debe al buen trabajo del medio sino a la audiencia que lo ha compartido con su entorno. Pero matiza que tampoco es intrínsecamente positivo, porque puede generarse tráfico que se mofe del contenido o que critique al medio. El click o el retuit puede tener dos direcciones.

El ejemplo de La Vanguardia y su estrategia de crecimiento es una evidencia para el resto de sus competidores. Elizari afirma que en Facebook está muy bien el panel de estadísticas gratuito con el que se puede monitorizar las páginas de la competencia. Admite que hay una página que está revolucionando todo y es la de La Vanguardia. ¿Su estrategia? Hacer todo lo que más interacción le reporta. "Da igual el coste que sea, el coste de marca". Y cita algunos contenidos banales con una interacción "brutal". En una semana "El País tenía 1'2, nosotros 0'6 y La Vanguardia 2'8 con cosas como éstas", expone.

Las redes tienen que servir "para que gente que no sabe quiénes somos o qué hacemos llegue a nosotros", enfatiza Rodríguez de El Confidencial. Considera que proyectos como Eldiario.es parten con ventaja con la comunión de sus 20.000 lectores, que incluso pagan para que el proyecto sobreviva. Analiza que los diferentes cambios de Facebook han creado campanas de opinión, lo que facilita que si se dispone de contactos o amigos en la red tengan predilección por ese diario. ¿Por qué? "Al final la gente en redes sociales tiende a compartir aquello que le ayuda a definirse ante los demás", concluye. A la representante de El País en estas entrevistas le gustaría disponer de una viralidad desglosada en función de los tipos de reacciones que despierta la red. Relata que sólo tiene la opción de hacerlo manualmente pero al carecer la API de la plataforma no trabajan en la extracción de datos. Éste diario utiliza la herramienta Spyke para rastrear contenidos de tendencia en redes sociales en tiempo real. El sistema lo emplea el equipo de redes y elabora tres informes al día que suscitan otras tantas reuniones a las 8, 14 y 20 horas, en las que se debate lo que sucede en las redes y las posibles apuestas del periódico.

También el Huffington mantiene reuniones periódicas para viralizar contenidos en las redes. Y en el caso de Facebook sobre todo cuando varía su algoritmo, lo que le obliga a marcar prioridades. El pago por darle mayor difusión a los contenidos también es una línea que se sigue por parte de buena parte de los grupos de comunicación, excepto en el caso de Joly, por el momento. Considera necesario disponer de una partida económica para aumentar la comunidad, pero ahora mismo no es factible. Aunque también hay matices. En Abc se justifica porque con esa fórmula se logra incrementar la base de seguidores. El Confidencial cuenta con un sistema automático que hace que determinadas piezas que alcanzan un determinado número de interacciones se promocionen solas. "Yo creo que es una aplicación que puede utilizar cualquiera", afirma Rodríguez. Pero también se recurre a la promoción pagada de contenidos en Facebook y señala que tiene como objetivo que, aquello que ya está funcionando bien, pueda presentarse ante audiencias nuevas, "que en principio son como la prueba del algodón".

El Español ha contratado a una agencia externa que vigila qué publicación funciona para invertir económicamente. Los responsables de redes no tienen poder decisorio. Como compensación, la empresa tampoco les puede marcar objetivo de crecimiento en Facebook, ya que tampoco manejan el presupuesto. El esfuerzo de promoción se ha dirigido a las secciones de estilo, la de motor y deportes. Los branded contents se pagan, pero porque van dentro del presupuesto de marketing. En función del balance del mes se decide por parte de este diario digital invertir más o menos para incrementar el tráfico.

Sobre la influencia de Facebook en los contenidos, desde el Huffington se admite que hay veces que se cede y en otras se trabaja con la hipótesis de que "con este Facebook tenemos que hacer 200.000, sino es que somos muy malos", detalla el interlocutor del medio digital. Pero el problema son algunos contenidos que él difunde y que sabe, de antemano, que son incompatibles con las normas de la red y son conscientes de que hay términos ligados por ejemplo al sexo donde funciona la autocensura.

El continuo cambio de los algoritmos es una preocupación latente por parte de la mayoría de los grupos consultados. Variaciones que suelen producirse con la frecuencia de una vez al mes. Si desaparecen de Google durante un tiempo con-

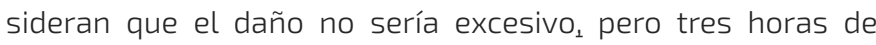
ausencia de Facebook se ve como algo difícil de recuperar. De ahí que las tarjetas amarillas, las advertencias que envía la red cada vez que algún medio incumple alguna norma del código deontológico que aplica la plataforma cuando se divulga alguna información, vídeo o imagen con contenidos violentos o sexuales se tengan muy en cuenta. "Sabes que Facebook te saca tarjeta amarilla: ¡Como vuelvas a publicar cosas así, a la siguiente te cerramos la cuenta! Las dos veces que nos ha salido eso es: ¡Por favor, los siguientes 40 días ni una tontería!", explica el subdirector del Huffington.

Uno de los asuntos más polémicos sobre los contenidos que circulan en Facebook son las fake news. En este sentido, el 
responsable de redes de El Confidencial considera que, a diferencia de otras redes como Twitter, al no incluir los llamados trending topic, tendencias que en principio sí se presentaron en Estados Unidos, pero que se suprimieron posteriormente al automatizar los equipos, provoca resultados negativos: "Ahora es un desastre porque se cuelan noticias falsas. Nosotros estamos pendientes", asegura Rodríguez de El Confidencial.

\subsection{El caso de Facebook Live}

Por otra parte, el nuevo modelo informativo demanda más contenido audiovisual. Pero exige una producción de material específico para la red. La esperanza para que este esfuerzo se traduzca en rentabilidad es que se pueda insertar publicidad. Corcuera confía en que esta hipótesis mejore económicamente el negocio y se materialice en un futuro no muy lejano. Si hay financiación directa la palabra rentabilidad aparece y también repercutiría en la mejora de contenidos. También José Manuel Rodríguez, de El Confidencial, se pronuncia a favor de la producción de contenidos nativos para estas redes, siempre que Facebook pague por los que se elaboran para su plataforma. En el Huffington confían igualmente en las posibilidades derivadas de Facebook Live cuando autorice insertar publicidad. Admiten que, a corto plazo, la producción de estos contenidos no aporta rentabilidad, pero al menos les sirve para difundir la marca. "Si yo consigo que de estos 800.000 que ven una información, 2.000 no me seguían y me empiezan a seguir, ya pincharán", afirma el subdirector del medio.

El fenómeno de Facebook Live es uno de los que más llama la atención en los últimos meses. La práctica totalidad de los grupos de comunicación españoles lo contemplan, ensayan y estudian todas sus posibilidades. El problema es que en la mayoría de los casos se producen con el móvil, la calidad es dudosa y en muchas ocasiones los informadores no están versados en este formato audiovisual. En El País distinguen diferentes tipologías de vídeo dentro de redes sociales. Por un lado $_{1}$ el vídeo nativo, el que se incrusta directamente dentro de la red social para que el usuario lo vea en sus perfiles, pero también comparte URL con vídeo. Y finalmente Facebook Live, una herramienta que están probando con profusión. El Mundo lanza estos vídeos desde un plató. Probaron con Periscope, pero sufrían muchos problemas técnicos que con esta alternativa de emisión en directo no se producen. También tienen estudiado que el impacto en la audiencia puede ser cien veces mayor.

El responsable de medios sociales de Abc afirma sobre Facebook Live que la calidad de lo que se emite por parte de unos medios concebidos para la producción de otro tipo de contenidos y con informadores no especializados en este tipo de productos, puede producir efectos perversos. Probaron el producto cuando se lanzó, analizaron los problemas técnicos de sonido e imagen y acotaron sus difusiones ante el riesgo de trasladar una sensación de amateurismo. Por su parte El País entiende que es una actuación que promueve directamente la plataforma. Bonachera y Morales, de El Español, admiten que lo que están haciendo ahora es seguir un poco la tendencia de Facebook Live. Eso se traduce en intentar producir el mayor número posible de videos en directo, dentro de las posibilidades de la redacción y con el obstáculo de la tecnología. De momento, emplean el móvil hasta disponer de cámaras. Le ven rentabilidad porque capta nuevas audiencias a las que enviar luego los enlaces y entienden que Facebook Live debe afrontarse como una estrategia a largo plazo.

Para El País, Facebook Live es estratégico, explica Pilar Millán:

Sí, como te digo es rentable porque tenemos que estar porque como bien sabes tenemos El País Video que es un área superestratégica del periódico y que en el último año ha crecido muchísimo, que nos ha traído muchas alegrías y que está formada por grandísimos profesionales y esa gran área del periódico se creó precisamente para entender mejor los consumos audiovisuales.

Desde El Confidencial aseguran que Facebook ha dado dinero a los medios como El País para que crearan directos. Pero advierten que hay que tener en cuenta dos cosas: los recursos de que dispone la redacción y si detrae de la elaboración de otras historias periodísticas. Y duda que ese esfuerzo compense sin más porque advierte un punto de cansancio en la audiencia. El riesgo es pasar de trabajar para el medio a hacerlo para la plataforma y el problema es la saturación del producto porque con tanta competencia es difícil la originalidad. El Mundo ha decidido transformar la sección de encuentros digitales para reconvertirla a Facebook Live. Sobre todo, utilizan muchos artistas. Creen que los directos que mejor funcionan son estos y los de a pie de calle.

Rodríguez, de El Confidencial, aprecia que los seguimientos en directo no son tantos como podría parecer en relación a la base de la página. Y dice que ese fenómeno también se produce en medios con muchos seguidores como la BBC. Y considera que el entretenimiento es el modelo que cosecha más éxitos con este formato. Además, defiende que el producto deba diferenciarse del que exhibe la televisión:

El algoritmo al final va ubicando las cosas y es difícil que todo el mundo tenga mucha visibilidad, porque todo el mundo está intentado entrar por el mismo sitio y se estrecha el filtro. Por otro lado, también tiene que ver con que el usuario va con el móvil -el 90\% de la gente que utiliza Facebook lo utiliza con móvil-, con una conexión o una tarifa de datos limitada, con lo cual la visión de vídeos le penaliza en ese aspecto y tiene que tener algo que te justifique realmente que se vea de forma masiva un directo. El reto era, sobre todo, intentar hacer algo que nos alejara de la televisión... Yo creo que prácticamente ninguno lo 
hemos conseguido.

El subdirector del Huffington profundiza en lo anterior. A veces el medio cree que el formato es el de la televisión y utiliza Facebook Live para transmitir mesas redondas, algo que duda tenga el mínimo interés para la audiencia. Asegura que El País hace tantos Facebook Live "porque pagan una millonada. Si a mí me pagaran, te hacía dos al día". Paradójicamente, han hecho pocos Facebook Live, pero su experiencia ha sido del todo positiva: "Como en cualquier redacción, hay muchas cosas que funcionan bien y de repente dejamos de hacer".

\section{Conclusiones}

Facebook se ha transformado en una plataforma necesaria e irrenunciable para las empresas periodísticas españolas. Pese a reconocer, como ya exponía Greenslade (2016) en The Guardian, que la plataforma puede imponer qué leer a los usuarios, que ha favorecido la profusión de noticias falsas y que incluso les obliga a practicar una autocensura con determinados contenidos, la consideran la red social a la que deben prestar más atención, entienden que es la apropiada para poder distribuir información y atraer nuevas audiencias, prioridades que ya advertía en su análisis de contenido sobre 41 medios europeos González y Reus (2103). Pero, además, defienden que también cumple con los requisitos para poder promocionar la imagen de marca.

La marca, la audiencia y el negocio son los tres vértices de la relación. Los principales medios españoles acuden a la red por su gran capacidad para viralizar los contenidos, lo que permite acceso a nuevos públicos ante los que trasladar el sello personal del medio al objeto de fidelizar a esos usuarios. Además, existe la firme esperanza de que los productos propios que demanda la plataforma, como los instant articles o Facebook Live, puedan rentabilizarse. Pero los planteamientos de partida colisionan con la realidad de las rutinas y la agenda. A pesar de esta dependencia que se ha generado, los medios consultados no disponen de personal destinado exclusivamente a trabajar en el entorno de Facebook. La interacción con las audiencias es mínima en todos los casos y aveces incluso se externaliza. No ha variado en la última década el escenario que ahora presentan las respuestas de los responsables de las redes con el que ofrecían estudios anteriores, como los de Hermida y Thurman (2008) sobre la prensa inglesa y su limitada conversación con la audiencia o el de Domingo et al (2008), que analizó la prensa europea y norteamericana.

Las únicas estrategias de los medios de referencia en España consisten en lanzar un número determinado de post al día, en general uno cada media hora, y, en mezclar, en algunos casos, noticias soft con hard, según las costumbres de la audiencia. Además, no sólo no cobran de Facebook sino que todos pagan por la promoción. Pero son los continuos cambios de algoritmo lo que se han convertido en una pesadilla para los periódicos con consecuencias en ocasiones demoledoras por la pérdida de audiencia y de comunidad, Bell (2016) atribuía a la red capacidad para devorar el mundo y en esa línea la desconfianza preside casi todas las relaciones de los medios consultados con la red. Sólo El País ve en Facebook un aliado y El Mundo se muestra también optimista al considerar que en la era digital no hay motivo para el temor a uno de sus grandes protagonistas, pero su alegato por el tráfico directo es incontestable.

\section{Agradecimiento}

Esta investigación se enmarca en el proyecto nacional "La influencia de la audiencia en la innovación periodística: riesgos y oportunidades" (CSO2015-64955-C4-3-R MINECO/FEDER).

\section{Referencias}

Bell, E. (2016). Facebook is eating the world. Columbia: Columbia Journalism review. Recuperado de https://www.cjr.org/analysis/ facebook_and_media.php

Bell, E., y Owen, T. (2017). How Silicon Valley reengineered journalism. Columbia: The Platform Press. Recuperado de https://www.cjr.org/ tow_center_reports/platform-press-how-silicon-valley-reengineered-journalism.php

Coca, J. R. (2009). Las redes sociales transforman los medios de comunicación. Madrid: Tendencias 21. Recuperado de http:// www.tendencias21.net/Las-redes-sociales-transforman-los-medios-de-comunicacion_a3410.html

Díaz, V. (2011). Mitos y realidades de las redes sociales, información y comunicación en las Sociedad de la Información. Prisma Social, Revista de Ciencias Sociales, (6), 340-366.

Domingo, D., Quandt, T ., Heinonen, A ., Paulussen, S ., Singer, J., y Vujnovic, M. (2008). Participatory journalism practices in the media and beyond: An international comparative study of initiatives in online newspapers. Journalism Practice, 2 (3), 326-342.

González, S., y Ramos, F. (2014). Las redes sociales en el ámbito periodístico: ¿cómo usan los medios europeos de referencia sus perfiles en Twitter y Facebook?. Comunicación y Hombre, (10), 37-52.

González, S., y Ramos, F. (2013). El uso periodístico de Facebook y Twitter: un análisis comparativo de la experiencia europea. Historia y Comunicación Social, 18, 419-433. doi:10.5209/rev_HICS.2013. v18.44253

Greenslade, R. (2016). Why Facebook is public enemy number one for newspapers, and journalism. The Guardian. Recuperado de https:// www.theguardian.com/media/greenslade/2016/sep/20/why-facebook-is-public-enemy-number-one-for-newspapers-and-journalism

Hermida, A., y Thurman, N. (2008). A Clash of Cultures. The integration of user-generated content within professional journalistic frameworks at British newspaper websites. Journalism Practice, 2(3), 343-56.

Kiss, J. (2016). A giant that may eat us. British Journalism Review, 27(3), 24-28. doi: 10.1177/0956474816668797

Merino, M., Lloves-Sobrado, B., y Pérez-Guerrero, A. (2013). La interacción de los usuarios en los perfiles de Facebook de la prensa española. Palabra Clave, 16(3), 842-872. doi: 10.5294/pacla.2013.16.3.7 
Noguera, J.M. (2010). Redes sociales como paradigma periodístico. Medios españoles en Facebook. Revista Latina de Comunicación Social, (65), 176-186.

Palomo, B., y Meso, K. (2014). El mito de la conversación con el medio: el caso de Facebook. En: González, J.E., y Valderrama, M. (coords.). Comunicación actual: redes sociales y lo 2.0 y 3.0. (pp. 453-468). Madrid: McGrawHill.

Pardo, P. (2017). Eli Pariser: El entretenimiento da más clics. El Mundo. Recuperado de http://www.elmundo.es/television/2017/06/19/ 594813f0e5fdea6a5a8b4593.html

Pozzi, S. (2017). Facebook gana un 71\% más en el segundo trimestre. El País. Recuperado de https://economia.elpais.com/ economia/2017/07/26/actualidad/1501086549_023562.html

Seco, R. (2017). Facebook apuesta por el periodismo de calidad. El País. Recuperado de https://elpais.com/tecnologia/2017/01/11/ actualidad/1484145535_207227.html

Segado, F., Díaz, J., y Soria, M. (2015). La viralidad de las noticias de Facebook. Factores determinantes. Telos: Cuadernos de comunicación e innovación, (100), 153-161.

Singer, J.B., Domingo, D., Heinonen, A., Hermida, A., Paulussen, S., Quant, T., Reich, Z., y Vujnovic, M. (2011). Participatory Journalism: Guarding Open Gates at Online Newspapers. Chichester, West Sussex: Wiley-Blackwell.

Somaiya, R. (2014). How Facebook Is Changing the Way Its Users Consume Journalism. The New York Times. Recuperado de https:// www.nytimes.com/2014/10/27/business/media/how-facebook-ischanging-the-way-its-users-consume-journalism.html
CV

Antonio Méndez. Profesor asociado del Departamento de Periodismo de la Universidad de Málaga. Doctor en Periodismo y Master en Dirección de Empresas Radiofónicas (Universidad Autónoma de Barcelona). Director del diario Málaga Hoy, posee más de 30 años de experiencia profesional como periodista en medios como EFE, Ser, El País y Grupo Joly. Sus líneas de investigación son la credibilidad informativa, la información oficial, el periodismo local y la radio generalista.

Agustín Rivera. Profesor asociado del Departamento de Periodismo de la Universidad de Málaga. Doctor en Periodismo, posee 24 años de experiencia profesional como periodista (redactor, jefe de sección, corresponsal en Tokio y enviado especial a 14 países de cuatro continentes) en medios de comunicación nacionales de referencia como El Confidencial, El Mundo y Diario 16. Actualmente, delegado del diario El Confidencial en Andalucía. Líneas de investigación: los cibermedios, el periodismo internacional y los géneros periodísticos.

Bella Palomo. Catedrática y Directora del Departamento de Periodismo de la Universidad de Málaga. Especializada en las áreas de cibermedios, audiencias activas, social media y rutinas periodísticas, es autora de un centenar de artículos y libros sobre estos temas. Ha sido consultora de multimedia y comunicación en la Universitat Oberta de Catalunya, y profesora visitante en las universidades de Washington, Rutgers, Miami (EUA), Shangai (China) y Federal de Bahía (Brasil). Actualmente es IP del proyecto "La influencia de la audiencia en la innovación periodística: riesgos y oportunidades" (CSO201564955-(4-3-R MINECO/FEDER).

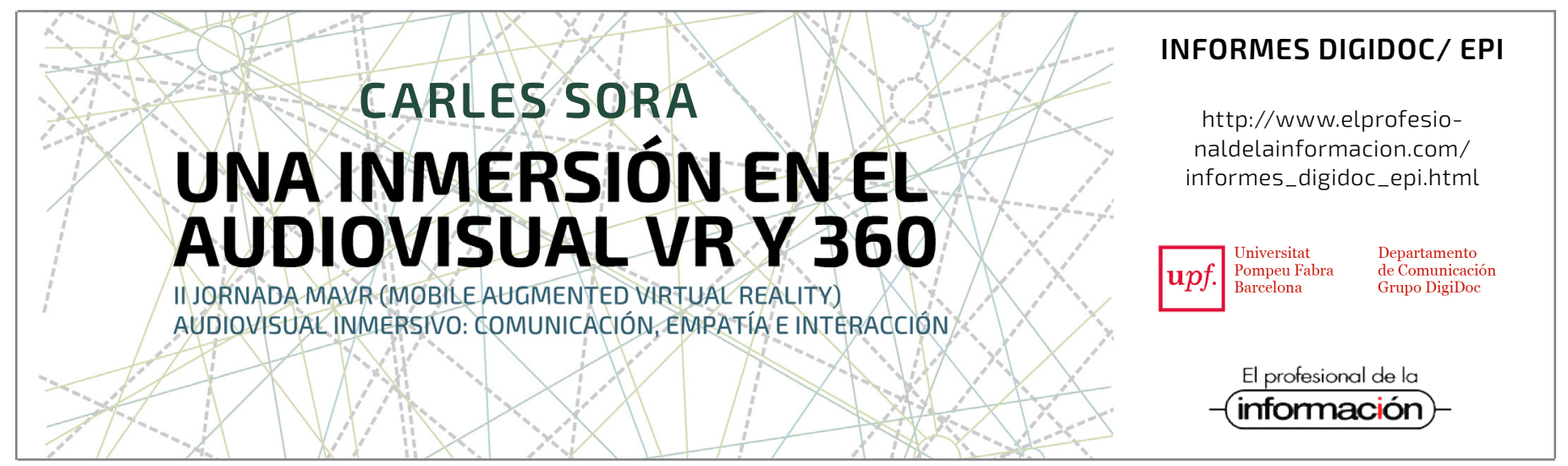

